\title{
Simultaneous quantitative analysis of four isothiazolinones and 3-iodo-2-propynyl butyl carbamate in hygienic consumer products
}

\author{
Jin Ju Heo', Un-Jung Kim ${ }^{1,2}$, Jeong-Eun $\mathrm{Oh}^{1^{+}}$ \\ ${ }^{1}$ Department of Civil and Environmental Engineering, Pusan National University, Busan 46241, Republic of Korea \\ ${ }^{2}$ Center for Environment, Health and Welfare Research, Korea Institute of Science and Technology (KIST), Seoul 02792, Republic of Korea
}

\begin{abstract}
In this study, extraction, clean-up and instrumental analytical conditions were optimized for identifying and quantifying methyl-, chloromethylbenzi-, octyl- isothiazolinone (MIT, CMIT, BIT, OIT) and 3-iodo-2-propynyl butyl carbamate (IPBC), widely used biocidal active ingredients in human hygiene consumer products. Accuracy of the developed ultrasonic assisted extraction method followed by HPLC/MS analysis for four isothiazolinones and IPBC ranged between $60.4 \%$ and $113 \%$ in various types of consumer product samples. Method detection limits ranged $0.011-0.034 \mathrm{mg} / \mathrm{kg}$ for wet wipes, $0.57-1.5 \mathrm{mg} / \mathrm{kg}$ for liquid detergent and 0.58-1.6 mg/kg for powder detergent. Wet wipes, powder and liquid detergents collected from local market in Korea were analyzed to demonstrate the applicability of the developed method. Even after the regulation of those compounds in wet wipes, CMIT, MIT and IPBC were still frequently detected from complementary wet wipes without brand labels which were distributed to customers at local retail shops and restaurants. The maximum observed concentrations of MIT and CMIT found in those complementary wet wipes were $70.2 \mathrm{mg} / \mathrm{kg}$ and $11.3 \mathrm{mg} / \mathrm{kg}$, respectively.
\end{abstract}

Keywords: Biocidal active ingredient, Consumer products, Detergent, UAE-HPLC/MS, Wet wipes

\section{Introduction}

Biocide is chemical and/or biological substance that destroys, deters, renders harmless, or exerts a controlling effect on any harmful organism. According to European Union Biocidal Products Directive (98/8/EC) [1, 2], biocides are possible to be classified into four categories (i.e., disinfectant/general biocide products, preservatives, pest control, and other biocide products) based on their intended usage. 3-iodo-2-propynyl butyl carbamate (IPBC) and four derivatives of isothiazolinone (e.g., Methylisothiazolinone (MIT), Chloromethylisothiazolinone (CMIT), 1,2-benzisothiazolinone (BIT) and 2-octyl-4-isothiazolin-3-one (OIT)) are disinfectant and preservative biocidal active ingredients that globally used in order to prevent growth of bacteria and mildew in various types of consumer products, such as cosmetics, hair and skin care products, cleaning agents, and fabric softeners [3-7]. There have been increasing concern regarding hidden environ- mental/health risk of pervasive and perpetual exposure to biocides that could occur during and after use of consumer products of containing biocides [4]. Biocides are known to be contact allergens to cause skin irritation and inhalation toxicity [2, 8-10]. In case of MIT, $\mathrm{LD}_{50}$ values from in vivo tests have been reported as 200 $\mathrm{mg} / \mathrm{kg}$ (dermal toxicity for rabbit), $105 \mathrm{mg} / \mathrm{kg}$ (oral toxicity for rat), and $0.33 \mathrm{mg} / \mathrm{L}$ (inhalation toxicity for rat) [7].

After environmental health crisis happened in Korea related with misuse of biocides (MIT/CMIT mixture) in the humidifier disinfectant, which recorded 78 casualties, including 36 infants due to fatal lung disease [11, 12], both compounds were listed as toxic substances on November 2012 by the Korea Chemicals Management Association (KCMA). However, public health risk of exposure to biocides is still concern as only limited types of consumer products were specified (i.e., fabric softener and wet wipe products) to prohibit the use of MIT/CMIT mixture in them [13]. The diversified exposure risk assessment and monitoring
This is an Open Access article distributed under the terms of the Creative Commons Attribution Non-Commercial License (http://creativecommons.org/licenses/by-nc/3.0/) which permits unrestricted non-commercial use, distribution, and reproduction in any medium, provided the original work is properly cited.
Received April 12, 2018 Accepted June 23, 2018

${ }^{\dagger}$ Corresponding author

Email: jeoh@pusan.ac.kr

Tel: +82-51-510-3513 Fax: +82-51-514-9574 
studies are, therefore, urgently demanded to establish appropriate regulation and to support management to control production and use of biocides [14]. As these compounds are applied in various types of consumer products, development of simple, accurate and sensitive analytical methods to quantify important biocidal active ingredients from complex sample matrix with covering different types of consumer products, is prerequisite to evaluate and manage potential risk of human exposure to such chemicals. So far, several analytical methods have been suggested to analyze isothiazolinones (e.g., UV-spectroscopy, GC-MS, HPLC-MS, and LC/MS/MS), but most of studies focused on environmental water (e.g., river and tap water) or sewage and much less information is available about analytical method of biocide in the consumer products [4-6, 15-17].

Therefore, in this study, analytical method for simultaneous identification and quantifications of MIT, CMIT, BIT, OIT and IPBC using high performance liquid chromatography/mass spectrometry (HPLC/MS) system with widened coverage of sample matrix was developed especially for determination of trace amount of biocides included in human hygiene biocidal products (Product type (PT) 01 class of the European Union Biocidal Products Directive [EU BPD]) comprising wet wipes and detergents [2]. The accuracy of the method was verified by analyzing matrix spike per each targeted sample type. The method was validated by analyzing 21 wet wipes and 8 detergents collected from local market in Korea. To the best of our knowledge, this is the first study to report on a simultaneous extraction and quantitative analysis by using HPLC/MS system for the four major isothiazolinones in various household products based on an internal standard method.

\section{Materials and Methods}

\subsection{Chemicals and Reagents}

MIT ( $\geq 94 \%$ ) and BIT (97\%) were purchased from Sigma-Aldrich (Steinheim, Germany). CMIT (99.9\%), OIT (99.9\%) and IPBC (99.7\%) were purchased from Ultra scientific (North Kingstown, USA). Two deuterated compounds of 2-octyl-4-isothiazolin-3one-d17 (OIT-d17, Toronto Research Chemicals, North York, Canada) and 3-(4-isopropylphenyl)-1,1-dimethylurea (Isoproturon-d6, Dr. Ehrenstorfer, Augsburg, Germany) were used as internal and recovery standards, respectively. The detailed information of target compounds was presented in the Table 1. Used organic solvents including acetone (Ac), acetonitrile (ACN), ethyl acetate (EtAC), methanol (MeOH), methyl tert-butyl ether (MTBE) were of HPLC grade while toluene (Tol) and methylene chloride (MC) were pesticide grade solvents that purchased from J.T. Baker (Philipsburg, NJ, USA). Supelclean ENVI-carb, ENVI-Chrom P cartridges (500 $\mathrm{mg}, 6 \mathrm{cc}$ ) and filtration tubes (empty cartridge, $6 \mathrm{cc}$ ) were purchased from Supelco (Bellefonte, USA). Oasis HLB cartridges $(200 \mathrm{mg}$, 6 cC) were purchased from Waters (Milford, USA). Aluminum oxide (98\% purity) was purchased from Sigma-Aldrich. The polytetrafluoroethylene (PTFE) syringe filter $(0.45 \mu \mathrm{m}$ pore size, 13 $\mathrm{mm}$ diameter) was purchased from Whatman (Maidston, UK). Standard solution for all target compounds was prepared in $\mathrm{MeOH}$.

\subsection{HPLC/MS System}

Target compounds were analyzed by high performance liquid chromatography (Agilent 1200 series HPLC; Agilent Technologies, Santa Clara, CA, USA) coupled with mass spectrometry (1100 MS; Agilent Technologies, Santa Clara, CA, USA) with C18 column (Hypersil gold; $4.6 \mathrm{~mm}$ x $250 \mathrm{~mm}, 5 \mu \mathrm{m}$; Thermo Fisher Scientific, Waltham, MA, USA) and Eclipse XDB C18 guard column (4.6 mm x $12.5 \mathrm{~mm}, 5 \mu \mathrm{m}$; Agilent Technologies). The injection volume was $10 \mu \mathrm{L}$ and positive electrospray ionization mode was used. The details of instrumental conditions, mobile phase, fragment masses for selective ion, and energy for quantification and identification are presented in Table S2.

\subsection{Consumer Products Collection}

The type and brand of consumer product samples were chosen based on published market analysis report of products with containing high content of biocidal active ingredients and domestic market survey of reporting approximate usage and production volume. The items selected for testing analytical method in this study were 21 wet wipes (WW), three powder type (PD) and five liquid type (LD) laundry detergents (including two fabric softeners, Table S3). To compare and optimize the extraction and purification conditions per sample type, one each powder and liquid detergent (PD 1 and LD 2), and three wet wipes (WW 1-3) were analyzed.

\subsection{Method Test Condition}

To optimize the analytical condition of biocides from consumer products, direct injection after dilution, solid phase extraction (SPE) and ultrasonic assisted extraction (UAE) were tested. All of the tested samples were initially fortified with known concen-

Table 1. Characteristics and Molecular Properties of the Target Compounds

\begin{tabular}{|c|c|c|c|c|c|c|}
\hline Compound & Formula & CAS No. & $\mathbf{M W}^{\mathrm{a}}$ & $\mathbf{m p}^{\mathbf{b}}\left({ }^{\circ} \mathrm{C}\right)$ & Solubility in water & $\log K_{\text {ow }}{ }^{c}$ \\
\hline MIT & $\mathrm{C}_{4} \mathrm{H}_{5} \mathrm{NOS}$ & $2682-20-4$ & 115.15 & $254-256$ & $960 \mathrm{~g} / \mathrm{L}^{\mathrm{d}}$ & $-0.49^{\mathrm{e}}$ \\
\hline CMIT & $\mathrm{C}_{4} \mathrm{H}_{4} \mathrm{CINOS}$ & $26172-55-4$ & 149.60 & - & water miscible ${ }^{\mathrm{d}}$ & $0.40^{\mathrm{e}}$ \\
\hline BIT & $\mathrm{C}_{7} \mathrm{H}_{5} \mathrm{NOS}$ & $2634-33-5$ & 151.19 & $154-158$ & Slightly soluble & $1.77^{\mathrm{f}}$ \\
\hline OIT & $\mathrm{C}_{11} \mathrm{H}_{19} \mathrm{NOS}$ & $26530-20-1$ & 213.34 & $<25$ & $500 \mathrm{mg} / \mathrm{L}^{\mathrm{d}}$ (at $25^{\circ} \mathrm{C}$ ) & $2.45^{\mathrm{e}}$ \\
\hline IPBC & $\mathrm{C}_{8} \mathrm{H}_{12} \mathrm{INO}_{2}$ & 55406-53-6 & 281.09 & $64-68$ & - & $4.44^{\mathrm{f}}$ \\
\hline
\end{tabular}

${ }^{\mathrm{a}} \mathrm{MW}$; Molecular Weight, ${ }^{\mathrm{b}} \mathrm{mp}$; melting point, ${ }^{\mathrm{C}} \log$ Kow; octanol/water coefficients, ${ }^{\mathrm{d}} \mathrm{http} / /$ Kischem.nier.go.kr, ${ }^{\mathrm{e} B e s t e r}$ et al., 2009,

${ }^{\mathrm{f}}$ Alvarez-rivera et al., 2012 
tration of internal standard (OIT-d17) before extraction. The recovery standard (Isoproturon-d6) was spiked into the final extractant prior to inject into HPLC/MS.

\subsubsection{Wet wipes}

For the direct injection of wet wipes, $0.5 \mathrm{~g}$ of the squeezed liquid from sample was 5 fold diluted with reagent water and vortexed for $30 \mathrm{~s}$. The $500 \mu \mathrm{L}$ homogenate was passed through syringe filter to remove any residual fibers and particles, transferred to a clean $2 \mathrm{~mL}$ vial and injected into the instrument. To configure appropriate SPE cartridge with the right conditioning and eluting solvent, experiment was performed with using various cartridge types, sample $\mathrm{pH}$ condition and by utilizing $\mathrm{Ac}, \mathrm{ACN}$ and $\mathrm{MeOH}$ as mobile phase referring from previous studies [3, 4]. Sample was $\mathrm{pH}$ adjusted to 2 and 7 , respectively, by adding $2 \mathrm{~N}$ hydrochloric acid and loaded onto each one of cartridge A (combination of $200 \mathrm{mg}$ HLB and $500 \mathrm{mg}$ C18 cartridge) and cartridge B (500 mg ENVI-Chrom P). Cartridge A was conditioned with $6 \mathrm{~mL}$ Ac: $\mathrm{MeOH}$ (1:1), $12 \mathrm{~mL} \mathrm{MeOH}$, and $20 \mathrm{~mL}$ reagent water. In case of cartridge $\mathrm{B}, 30 \mathrm{~mL}$ ACN:MeOH (1:1) and $30 \mathrm{~mL}$ reagent water was used. The eluting solvent for cartridge A was applied with $20 \mathrm{~mL}$ Ac:MeOH (1:1), while $30 \mathrm{~mL}$ ACN:MeOH (1:1) was applied for cartridge B. In case of UAE, 10\% Tol in MC was tried with modification from Lin et al. (2010) [18]. One gram of liquid from wet wipes was thoroughly mixed with $14 \mathrm{~mL}$ of extraction solvent and vortexed for $1 \mathrm{~min}$. The homogenized sample was extracted by a $10 \mathrm{~min}$ sonication ( 200 watt, $15^{\circ} \mathrm{C}$ ), shaken for $5 \mathrm{~min}$ at $300 \mathrm{rpm}$ at $25^{\circ} \mathrm{C}$, then were centrifuged at $4,000 \mathrm{rpm}$ for $10 \mathrm{~min}$ to separate organic phase. The extractant was then passed through a $690 \mathrm{mg}$ activated alumina powder packed cartridge for purification.

\subsubsection{Detergent}

For the detergent, SPE for both powder and liquid samples was applied based on combination of the successful extraction condition of CMIT, OIT and BIT from water samples reported with some modifications [3, 4]. Briefly, two different cartridges (200 mg HLB and $500 \mathrm{mg}$ ENVI-Chrom P) were preconditioned with MC (12 mL), MeOH (12 mL), and reagent water $(12 \mathrm{~mL})$, consecutively. Each $0.5 \mathrm{~g}$ of sample was diluted (1/50 fold) from the raw material by pure water, loaded onto each of two cartridges, respectively, and eluted with $12 \mathrm{~mL}$ of $\mathrm{MC}$. The identical operating conditions used for wet wipes were applied for optimizing UAE condition for detergents, while experiments were performed to select an optimal extracting solvent among $\mathrm{MeOH}$, EtAC, MTBE, $\mathrm{MC}$, and their mixtures based on the solubility for the target compounds. $0.5 \mathrm{~g}$ of samples were mixed with $10 \mathrm{~mL}$ of each testing solvent, extracted three times, and finally merged together in a tube to fit a final volume of $30 \mathrm{~mL}$.

\subsection{Quality Assurance/Quality Control}

A nine-point standard curve covering concentration range of $20-1,500 \mathrm{ng} / \mathrm{mL}$ of the five target biocides was prepared in $\mathrm{MeOH}$. All calibration standards contained $500 \mathrm{ng} / \mathrm{mL}$ of the internal standard (OIT-d17) and $200 \mathrm{ng} / \mathrm{mL}$ of the recovery standard (Isoproturon-d6). The middle stage $(250 \mathrm{ng} / \mathrm{mL})$ calibration standard was checked every $12 \mathrm{~h}$ as an initial calibration verification during analysis. The same standard solution was regularly injected for checking the signal stability from HPLC/MS to avoid any inter-batch difference of sensitivity during this study. The extraction efficiency of each tested method was estimated by calculating recovery rate of tested consumer product samples. The method detection limit (MDL) and accuracy were verified by analyzing matrix spike samples which contain $100 \mathrm{ng} / \mathrm{mL}$ and $250 \mathrm{ng} / \mathrm{mL}$ of target biocides, respectively. The method detection limits (MDLs, Table S1) were determined by multiplying by 3.14 and the standard deviation of the seven matrix spike replicates per sample type. To assess potential background contamination, procedural blanks ( $\mathrm{n}=4$ per batch) and instrument blanks ( $\mathrm{n}=7-10$ per batch) were analyzed in every experimental batch.

\section{Results and Discussion}

\subsection{Instrumental Analytical Parameter Set Up}

C18 column was chosen for this study on the basis of the previously reported analytical conditions of targeting MIT and CMIT [6, 18, 19]. Based on the preliminary chromatographic separation (SI 1) with solvent $\mathrm{A}(50 \% \mathrm{MeOH}$ in water) and $\mathrm{B}(\mathrm{MeOH})$, gradient program condition (i) was developed that started with 50\% B for $14 \mathrm{~min}$ (flow rate; $0.35 \mathrm{~mL} / \mathrm{min}$ until $11 \mathrm{~min}$ then increased as $0.4 \mathrm{~mL} / \mathrm{min}$ until $14 \mathrm{~min}$ ), increased to $90 \%$ B for $25 \mathrm{~min}$, and increased to $100 \% \mathrm{~B}$ until $35 \mathrm{~min}$ (flow rate; $0.5 \mathrm{~mL} / \mathrm{min}$ ). All target compounds were clearly separated as shown in Fig. 1(a). There were no critical variations in the peak abundance during the five sequential injections, showing a standard deviation within $2 \%$. The optimal ionic strength of polar solvent (solvent A in the gradient program) was investigated by comparing the peak

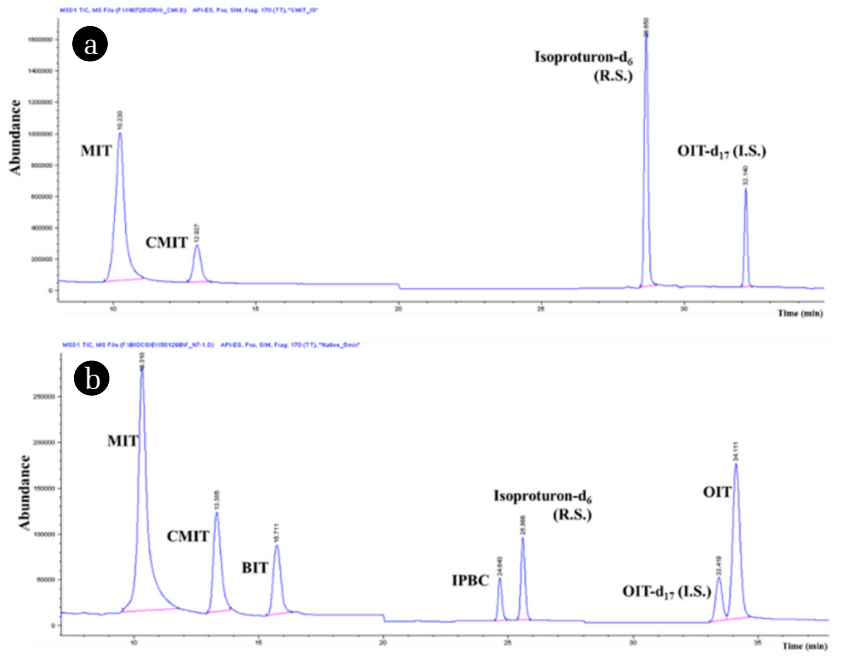

Fig. 1. Total ion chromatogram (TIC) of five target biocides $(500 \mathrm{ng} / \mathrm{mL})$ according to solvent gradient; (a) CMIT/MIT mixture- the gradient of the two following mobile phases was initiated with $50 \%$ B $(\mathrm{MeOH})$ for 14 min, increased to $90 \% \mathrm{~B}$ until $25 \mathrm{~min}$ and increased $100 \%$ B until 35 min (b) Five biocides mixture- the gradient system as follow; 0 min to $11 \mathrm{~min} 50 \%$ B, increased to $70 \%$ $\mathrm{B}$ until $15 \mathrm{~min}$ and kept up to $38 \mathrm{~min}$. 


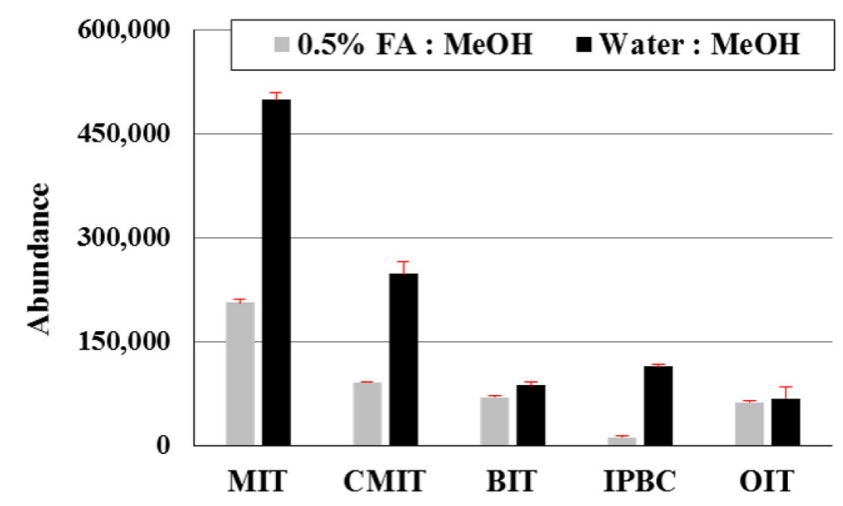

Fig. 2. Quantitative ion peak abundance with $0.5 \%$ formic acid-MeOH and water-MeOH as mobile phase.

abundances between pure water and $0.5 \%$ formic acid for the condition (i). The peak abundance of MIT and CMIT was 2-3 times higher with pure water than the $0.5 \%$ formic acid introduced condition as shown in Fig. 2.

The optimized mobile phase condition for MTT and CMIT was further expanded to analyze BIT, IPBC, and OIT simultaneously after a minor modification to produce HPLC condition (ii); $50 \%$ B for $11 \mathrm{~min}(0.35 \mathrm{~mL} / \mathrm{min})$, increased to $70 \%$ B until $15 \mathrm{~min}$ ( $0.4 \mathrm{~mL} / \mathrm{min})$, and then held until $38 \mathrm{~min}(0.6 \mathrm{~mL} / \mathrm{min})$. The total ion chromatogram (TIC) of condition (ii) was shown in Fig. 1(b). All target compounds were well separated, with showing capacity factor (a) of 1.02-3.31 between each compound. Therefore, all test samples for optimizing pre-treatment methods were analyzed by condition (i) for MIT/CMIT and continued to be analyzed by condition (ii) to conduct simultaneous analysis of five biocides. The typical HPLC/MS ion chromatogram of each type of sample was shown in Fig. S1.

\subsection{Extraction and Clean-up}

The experimental methods were firstly screened out by comparing reported extraction recovery from previous literatures. Mostly, liquid samples (i.e., tap and river water, sewage and wastewater) were pre-treated by SPE [3, 4, 15], while solid samples (i.e., sludge, sediment, cosmetics, and paper for food packaging) were pre-treated by UAE $[15,17,18]$. Therefore, these two methodologies were primarily considered and optimized by manipulating various experimental parameters to achieve high extraction efficiency and purification of target biocides from various types of consumer products. All test samples were spiked with $1,000 \mathrm{ng} / \mathrm{mL}$ of MIT/CMIT and $500 \mathrm{ng} / \mathrm{mL}$ of internal standard (OIT-d17) prior to extraction and were spiked with $200 \mathrm{ng} / \mathrm{mL}$ of recovery standard (Isoproturon-d6) before injection. The final extractants of samples were filtered with a polytetrafluoroethylene (PTFE) syringe filter $(0.45 \mu \mathrm{m})$, concentrated under a gentle stream of nitrogen, and reconstituted with $1 \mathrm{~mL}$ of $\mathrm{MeOH}$. The detailed procedure, parameters, and results were described below per each sample type.

\subsubsection{Detergent}

Table 2 shows recoveries of MIT and CMIT from detergent samples according to tested method. SPE method was tried by using HLB and ENVI-Chrom P cartridges. Due to high viscosity after concentration, SPE method was not considered any further for detergents. Four different organic solvents (MeOH, EtAC, MTBE, and MC) were chosen to optimize the extraction conditions for UAE. MC extraction was preferred to perform instrumental analysis because the others contained viscous interferences in the final extractant. The recoveries of MT and CMIT extracted with MC were below 40\% (MIT; $19-22 \%$, CMIT; 28-39\%). To minimize ion suppression due to sample matrix, ENVI-carb cartridge (500 mg, 6 cc) and activated alumina cartridge ( $2 \mathrm{~g}$ alumina and $1 \mathrm{~g}$ sodium sulfate) was connected in order and preconditioned with $12 \mathrm{~mL} \mathrm{MC} \mathrm{prior}$ to pass through the extractant. After purification, the extraction efficiency of both compounds was improved (MT: 80.0-80.9\%, CMIT; 80.2-99.7\%; Table 3). From Alvarez-Rivera et al. (2012), recovery of MIT, CMIT, BIT, and OIT with matrix solid-phase dispersion (MSPD)-LC/MS/MS was reported to range from 59.1 to $86.3 \%$ for liquid dishwasher detergent and $56.3-90.9 \%$ for other consumer products such as shampoo, baby care products, hand cream, bath gel, and surface cleaning cream [5].

The final extraction method selected for the detergent samples was UAE coupled with purification using a combination of alumina and ENVI-Carb cartridges. The detailed procedure was as follows: $50 \mathrm{mg}$ of detergent sample (20 mg for fabric softener) was diluted into $25 \mathrm{~mL}$ water and the whole mixture solution was extracted by using $25 \mathrm{~mL}$ of MC:EtAC (9:1) for $10 \mathrm{~min}$ sonication (200 watt, $\left.15^{\circ} \mathrm{C}\right)$ and shaking for $5 \mathrm{~min}\left(300 \mathrm{rpm}, 25^{\circ} \mathrm{C}\right)$. The extract was centrifuged at 4,000 rpm for $10 \mathrm{~min}$ to remove water phase then final extracts were passed through ENVI-carb cartridges and activated alumina cartridges in order. Excess solvent was evaporated near dryness under nitrogen stream and reconstituted with $1 \mathrm{~mL}$ of $\mathrm{MeOH}$ that was fortified with a recovery standard prior to inject to HPLC/MS.

\subsubsection{Wet wipes}

In case of wet wipes, direct injection after dilution, SPE and UAE method were tried. The recovery results for MIT and CMIT were shown in Table 3. The average recovery of direct injection method after 5 times dilution of original sample was 35\% (19-50\%) for MTT and $128 \%$ (50-170\%) for CMIT, respectively. Over than $45 \%$ of relative standard deviation (RSD) was observed when the method was applied to different brands of wet wipes. SPE was tested with different $\mathrm{pH}$ conditions ( $\mathrm{pH} 2$ and 7 ) and cartridge types (combination of HLB with C18 and ENVI-Chrom P). Overall recovery was low regardless of the type of cartridge and $\mathrm{pH}$ condition (MIT, 8-18\%; CMIT, 22-39\%).

UAE method was tried with $10 \%$ Tol in MC to extract MIT and CMIT from wet wipes. The extracts were passed through an activated alumina cartridge (690 $\mathrm{mg}$ ) that was preconditioned by $12 \mathrm{~mL} \mathrm{MC}$. The recovery of MT and CMIT was lot improved to $100 \%(87-110 \%)$ and $90 \%$ (77-102\%), respectively, and the standard deviation of recoveries was below $15 \%$ for all three types of test sample. The extraction efficiency of MIT or CMIT from wet wipes were not reported so far but compared with water sample results including tap water [3], distilled water [4], river water and sewage effluents [6] using SPE and large-volume direct injection method (Table S4). The reported recoveries of CMIT were $49-98 \%$ and the recoveries of MIT showed wide range (5-92\%). 
Table 2. The Recoveries of MIT and CMIT in Different Pretreatment Methods for Liquid and Powder Type Detergent (Unit: \%)

\begin{tabular}{|c|c|c|c|c|c|c|c|}
\hline & \multicolumn{2}{|c|}{ SPE method } & \multicolumn{5}{|c|}{ UAE method } \\
\hline & \multirow{2}{*}{ HLB } & \multirow{2}{*}{ Envi-chrom $^{\text {e }}$} & \multicolumn{5}{|c|}{ Extracting Solvent } \\
\hline & & & $\mathrm{MeOH}^{\mathrm{a}}$ & ETAC $^{\mathbf{b}}$ & MTBE $^{\mathrm{c}}$ & MC $^{\mathrm{d}}$ & MC:EtAc $(9: 1)^{\mathrm{e}}$ \\
\hline \multicolumn{8}{|c|}{ Liquid type detergent } \\
\hline MIT & $N A^{f}$ & NA & NA & NA & NA & 19 & 80 \\
\hline CMIT & NA & NA & NA & NA & NA & 38.5 & 99.7 \\
\hline \multicolumn{8}{|c|}{ Powder type detergent } \\
\hline MIT & NA & NA & NA & NA & NA & 22 & 80.9 \\
\hline CMIT & NA & NA & NA & NA & NA & 28 & 80.2 \\
\hline
\end{tabular}

${ }^{\mathrm{a}} \mathrm{MeOH}$; Methanol, ${ }^{\mathrm{b}} \mathrm{ETAC}$; Ethyl acetate, ${ }^{\mathrm{C}} \mathrm{MTBE}$; Methyl-tert-butyl ester, ${ }^{\mathrm{d}} \mathrm{MC}$; Methylene chloride, ${ }^{\mathrm{e}}$ purification; Extracts were passed from ENVI-carb (500 mg, $6 \mathrm{cc}$ ) to activated alumina cartridge (packed 2 g alumina and 1 g sodium sulfate) for purification, ${ }^{\mathrm{f}} \mathrm{NA}$; Not available to analyze due to the effect of impurities

Table 3. The Recoveries (RSD) of MIT and CMIT in Different Pretreatment Methods for Wet Wipes (Unit: \%)

\begin{tabular}{|c|c|c|c|c|c|c|}
\hline & \multirow{3}{*}{ Direct injection } & \multicolumn{4}{|c|}{ SPE } & \multirow{3}{*}{ UAE } \\
\hline & & \multicolumn{2}{|c|}{ HLB +C18 } & \multicolumn{2}{|c|}{ ENVI-chrome $P$} & \\
\hline & & pH 2 & pH 7 & pH 2 & pH 7 & \\
\hline MIT & $35(45)$ & $8(0.7)$ & $7(0.76)$ & $18(1.6)$ & $15(4.8)$ & $100(12)$ \\
\hline CMIT & $128(53)$ & $22(1.1)$ & $37(6.0)$ & $23(0.03)$ & $39(3.1)$ & $90(14)$ \\
\hline
\end{tabular}

Wittenberg et al. (2015) recently reported MIT and CMIT recoveries of 103 and 97\%, respectively, using the solvent extraction method which is comparable level of recovery with our results [20]. The flow chart of pre-treatment method and extract ion chromatograms of each sample were shown in Fig. S1 and Fig. S2.

\subsubsection{Method validation.}

The coefficient of determination $\left(\mathrm{R}^{2}\right)$ for the calibration curves (20-1,500 ng/mL) was above 0.99 for all target analytes as shown in Fig. S3. The relative standard deviation (RSD, \%) of initial calibration verification was within $10 \%$ after $20 \mathrm{~h}$. The recoveries of five biocides in wet wipes $(\mathrm{n}=5, \mathrm{WW}$ ), and liquid ( $\mathrm{n}=$ 5, LD 4) and powder ( $\mathrm{n}=5$, PD 2) detergent samples, were 80.3-113\%, 62.4-103\%, and 60.4-94.3\%, respectively (Table S1, Table S4). There are no studies that reported results for the target isothiazolinones from same types of samples that analyzed in this study at this moment so overall reported experimental recoveries for various sample types (i.e., household and cosmetic products, adhesives, river and tap water, distilled water) were compared with our result. The reported recoveries were 5-103\% for MIT, 49-103\% for CMIT, 15-103\% for BIT, $89 \%$ for IPBC, and $85-99 \%$ for OIT which were similar to or lower than the recovery values by using our method [3-5, 20, 21].

The MDLs in wet wipes were $0.011-0.034 \mathrm{mg} / \mathrm{kg}$; liquid detergent, 0.57-1.5 mg/kg; and powder detergent, 0.58-1.6 mg/kg and compared with the reported MDLs from various type of sample from previous literatures (Table S4) [5]. Alvarez-Rivera et al. (2012) reported MDLs of 0.0066-0.060 ppm for four biocides (MT, CMT, BIT, and OIT) in household products [5]. It was 2 to 5 times higher than our MDL for wet wipes and at maximum around 30 times higher (except OIT) than our MDL for the detergents.
The MDLs were approximately 10-10,000 times lower than most of guideline values from many countries such as EU [22], Japan [23], Denmark for MT (100 mg/kg) and CMIT (15 mg/kg) in wet wipes and fabric softener samples $[14,24]$. Cosmetic products are permitted to contain up to $100 \mathrm{ppm}$ of MIT, and $15 \mathrm{ppm}$ of CMIT/MIT (3:1 mixture) in the EU [22], Japan [23], Denmark [24]. Other isothiazolinone compounds such as BIT and OIT are permitted to contain up to $500 \mathrm{ppm}$ in cosmetics, detergents, cleaning products, and paints. Therefore, the proposed method is highly capable of analyzing trace levels of biocides from consumer products.

\subsection{Korean Market Consumer Products}

To confirm the applicability of the optimized analytical method of biocides developed in this study, 29 consumer products from the Korean market were analyzed for five biocides and their concentration profile was shown in Table 4 and Table S5. Among three powder type detergents, OIT (38.6 mg/kg) was detected in only one sample. In case of five liquid type detergent samples, MIT, BIT, and OIT were detected in the range of $2.59-99.0 \mathrm{mg} / \mathrm{kg}$ and their detection frequency (DF) was less than $40 \%$. Target biocides except OIT were found lower than MDL (0.0005-0.0054 mg/kg) in wet wipes used for personal care. However, MT (100\%), CMIT (69\%), and IPBC (77\%) were detected with higher frequency in wet wipes that were collected from local restaurants, which are usually given to customers as sales promotion. The observed concentrations of MIT (0.03-70.2 mg/kg) and CMIT (ND-11.3 mg/kg) in complementary wet wipes without brand labels were still lower than the Korean guideline value (MIT, $100 \mathrm{mg} / \mathrm{kg}$; CMIT, $15 \mathrm{mg} / \mathrm{kg}$, [14]) but it found to be possible that human exposure for biocides 
Table 4. Observed Concentration Levels $(\mathrm{mg} / \mathrm{kg}$ ) of Five Biocides in Common Products

\begin{tabular}{|c|c|c|c|c|c|}
\hline & MIT & CMIT & BIT & IPBC & OIT \\
\hline \multicolumn{6}{|c|}{ Wet wipes for sales $(n=8)$} \\
\hline Mean & - & - & - & - & 0.096 \\
\hline Detection Frequency (DF) & $0 / 8$ & $0 / 8$ & $0 / 8$ & $0 / 8$ & $2 / 8$ \\
\hline \multicolumn{6}{|c|}{ Wet wipes in local restaurants $(n=13)$} \\
\hline Mean & 30.3 & 2.97 & - & 0.58 & - \\
\hline $\mathrm{DF}$ & $13 / 13$ & $9 / 13$ & $0 / 13$ & $10 / 13$ & $0 / 13$ \\
\hline \multicolumn{6}{|c|}{ Powder type detergent $(n=3)$} \\
\hline Mean & - & - & - & - & 38.6 \\
\hline $\mathrm{DF}$ & $0 / 3$ & $0 / 3$ & $0 / 3$ & $0 / 3$ & $1 / 3$ \\
\hline \multicolumn{6}{|c|}{ Liquid type detergent $(n=5)$} \\
\hline Mean & 2.59 & - & 9.9 & - & 99.0 \\
\hline $\mathrm{DF}$ & $1 / 5$ & $0 / 5$ & $2 / 5$ & $0 / 5$ & $1 / 5$ \\
\hline
\end{tabular}

through use of the complementary products even after the banning of production and sales of major consumer products. The samples analyzed in this study are not cosmetics but we can consider the leave-on effect from wet wipes as customers usually use wet wipes to clean their hands instead of washing hands with water. The maximum concentration of MIT/CMTT allowed in Europe was $15 \mathrm{ppm}$ in cosmetics while in the United States, the concentrations allowed are set to $15 \mathrm{ppm}$ and $7.5 \mathrm{ppm}$ in rinse-off products and leave-on cosmetics, respectively [25]. Also, considering these compounds are widely used preservatives in many different types of consumer products which could be another source of biocides to human exposure [26], it should not be underestimated that the potential exposure risk derived from the observed amounts of MTT and CMIT in the consumer products off the regulation to human.

From previous study in Spain, the levels of MT were reported in laundry detergent and softener that were similar to the levels observed in this study: 1.18 and $2.71 \mathrm{ppm}(\mathrm{w} / \mathrm{w})$, respectively [5]. However, the level of BIT (average concentration, $150 \mathrm{ppm}$ ) in cleaning products was higher than our study. In the US, the level of MIT/CMIT in cosmetic products, such as shampoo/conditioner and skin care products, was in the range of ND-89.6 mg/kg and ND-10.3 mg/kg, respectively, and the detection frequencies of MIT and CMIT were 95\% and 29\%, respectively [20].

In light of widespread usage of MIT/CMIT and other biocides in personal care products, skin sensitization in the general population is drawing rising attention. Recently, analytical method for targeting metabolites of isothiazolinones in human urine recently reported [27-29]. To fill the gap between wide occurrence of isothiazolinones and human health risk derived from exposure to biocides, further studies of monitoring human specimens with matched information about life style (e.g. consumer product usage and frequency) questionnaires will be recommended.

\section{Conclusions}

This study presented a simple simultaneous analytical method for five emerging concern biocidal active ingredients that are frequently used in consumer products, with a special focus on MIT and CMIT in wet wipes and detergents. Of the three pre-treatment methods we tested, UAE had the highest extraction efficiency with MC:EtAC (9:1) for detergents and with MC:Tol (9:1) for wet wipes that were cleaned up with alumina cartridge. This method was confirmed to be applicable for the simultaneous analysis of BIT, IPBC, and OIT from diverse in use consumer products. The MDL for five target biocides of proposed analytical method is sufficient to analyze target compounds lower than guideline values. The observed concentration level of MIT, CMIT, and IPBC in complementary wet wipes without brand labels was surprisingly high and detected from more than $60 \%$ of collected samples even after regulation. Therefore, human exposure to isothiazolinone class biocides is highly suspected and further researches are strongly recommended for continuous monitoring of consumer products to assess human health risk related with exposure through consumer products usage.

\section{Acknowledgments}

This study was supported by grant Korean Ministry of Environment (MOE) through "The Environment Health Action Program (ARQ201303083)”.

\section{References}

1. "Biocide”. Wikipedia [Internet]. c2016 [cited 13 March 2016]. Available from: http://www.en.wikipedia.org/wiki/Biocide.

2. Cincinnati O. NEPIS Document display [Internet]. Available from: http://www.nepis.epa.gov/Exe/ZyNET.exe (accessed November 15, 2015).

3. Rafoth A, Gabriel S, Sacher F, Brauch HJ. Analysis of isothiazolinones in environmental waters by gas chromatography-mass spectrometry. J. Chromatogr. A. 2007;1164:74-81. 
4. Bester K, Lamani X. Determination of biocides as well as some biocide metabolites from facade run-off waters by solid phase extraction and high performance liquid chromatographic separation and tandem mass spectrometry detection. J. Chromatogr. A. 2010;1217:5204-5214.

5. Alvarez-Rivera G, Dagnac T, Lores M, et al. Determination of isothiazolinone preservatives in cosmetics and household products by matrix solid-phase dispersion followed by high-performance liquid chromatography-tandem mass spectrometry. J. Chromatogr. A. 2012;1270:41-50.

6. Speksnijder P, van Ravestijn J, de Voogt P. Trace analysis of isothiazolinones in water samples by large-volume direct injection liquid chromatography tandem mass spectrometry. $J$. Chromatogr. A. 2010;1217:5184-5189.

7. Kahrilas GA, Blotevogel J, Stewart PS, Borch T. Biocides in hydraulic fracturing fluids: A critical review of their usage, mobility, degradation, and toxicity. Environ. Sci. Technol. 2015;49:16-32.

8. Basketter DA, Rodford R, Kimber I, Smith I, Wahlberg JE. Skin sensitization risk assessment: A comparative evaluation of 3 isothiazolinone biocides. Contact Dermatitis 1999;40:150-154.

9. Boyapati A, Tam M, Tate B, Lee A, Palmer A, Nixon R. Allergic contact dermatitis to methylisothiazolinone: exposure from baby wipes causing hand dermatitis. Australas. J. Dermatol. 2013;54:264-267.

10. Fewings J, Menné T. An update of the risk assessment for methylchloroisothiazolinone/methylisothiazolinone (MCI/MI) with focus on rinse-off products. Contact Dermatitis 1999;41:1-13.

11. c2015 [cited 21 November 2015]. Available from: http://www. pressian.com/news/article.html?no=5857 [Internet].

12. Park DU, Friesen MC, Roh HS, et al. Estimating retrospective exposure of household humidifier disinfectants. Indoor Air. 2015;25:631-640.

13. c2015 [cited 21 November 2015]. Available from: http://www. kcma.or.kr/chemicals/chemicalss1_1.asp [Internet].

14. c2015 [cited 21 November 2015]. Available from: http://www.kats.go.kr/content.do?cmsid=239\&cid=10961\&m ode $=$ view $[$ Internet $]$.

15. Chen ZF, Ying GG, Lai HJ, et al. Determination of biocides in different environmental matrices by use of ultra-high-performance liquid chromatography-tandem mass spectrometry. Anal. Bioanal. Chem. 2012;404:3175-3188.

16. Lundov MD, Kolarik B, Bossi R, Gunnarsen L, Johansen JD. Emission of isothiazolinones from water-based paints. Environ. Sci. Technol. 2014;48:6989-6994.

17. Frauen M, Steinhart H, Rapp C, Hintze U. Rapid quantification of iodopropynyl butylcarbamate as the preservative in cosmetic formulations using high-performance liquid chromatographyelectrospray mass spectrometry. J. Pharmaceut. Biomed.
2001;25:965-970.

18. Lin QB, Wang TJ, Song H, Li B. Analysis of isothiazolinone biocides in paper for food packaging by ultra-high-performance liquid chromatography-tandem mass spectrometry. Food. Addit. Contam. Part A. 2010;27:1775-1781.

19. Singer H, Jaus S, Hanke I, Lück A, Hollender J, Alder AC. Determination of biocides and pesticides by on-line solid phase extraction coupled with mass spectrometry and their behaviour in wastewater and surface water. Environ. Pollut. 2010;158: 3054-3064.

20. Wittenberg JB, Canas BJ, Zhou W, Wang PG, Rua D, Krynitsky AJ. Determination of methylisothiazolinone and methylchloroisothiazolinone in cosmetic products by ultra high performance liquid chromatography with tandem mass spectrometry. J. Sep. Sci. 2015;38:2983-2988.

21. Rosero-Moreano M, Canellas E, Nerín C. Three-phase hollow-fiber liquid-phase microextraction combined with HPLC-UV for the determination of isothiazolinone biocides in adhesives used for food packaging materials. J. Sep. Sci. 2014;37:272-280.

22. c2016 [cited 12 December 2016]. Available from: http://www. sveff.se/files/2015/04/CEPE-guidance-on-labelling-with-biocidesskin-sensitizers-final-28-Oct-14.pdf [Internet].

23. Burnett CL, Bergfeld WF, Belsito DV, et al. Final report of the safety assessment of methylisothiazolinone. Int. J. Toxicol. 2010;29:187S-213S.

24. Krzywkowski K, Tønning K, Schwensen JF, Johansen JD. Survey and exposure assessment of methylisothiazolinone in consumer products. The Danish Environmental Protection Agency; 2015. p. 18-71.

25. Cuesta L, Silvestre JF, Toledo F, Ballester I, Betlloch I. Not detected by the baseline series of the Spanish group. Contact Dermatitis 2010;62:250-251.

26. González-Muñoz P, Conde-Salazar L, Vañó-Galván S. Allergic contact dermatitis caused by cosmetic products. Actas Dermo-Sifiliográficas (English edition) 2014;105:822-832.

27. Schwensen JF, Menné T, Andersen KE, Sommerlund M, Johansen JD. Occupations at risk of developing contact allergy to isothiazolinones in Danish contact dermatitis patients: Results from a Danish multicentre study (2009-2012). Contact Dermatitis 2014;71:295-302.

28. Schettgen T, Bertram J, Kraus T. Quantification of N-methylmalonamic acid in urine as metabolite of the biocides methylisothiazolinone and chloromethylisothiazolinone using gas chromatography-tandem mass spectrometry. J. Chromatogr. B. 2017;1044-1045:185-193.

29. Schettgen T, Kraus T. Urinary excretion kinetics of the metabolite N-methylmalonamic acid (NMMA) after oral dosage of chloromethylisothiazolinone and methylisothiazolinone in human volunteers. Arch. Toxicol. 2017;91:3835-3841. 